(C) [2008] IEEE. Reprinted, with permission, from [K. B. Dossou, L. C. Botten, S. Mahmoodian, R. C. McPhedran, C. G. Poulton, A. A. Asatryan and C. Martijn de Sterke, Modes of composite defects in 2D photonic crystals, Opto-Electronics and Communications Conference, 2008 and the 2008 Australian Conference on Optical Fibre Technology. OECC/ACOFT 2008]. This material is posted here with permission of the IEEE. Such permission of the IEEE does not in any way imply IEEE endorsement of any of the University of Technology, Sydney's products or services. Internal or personal use of this material is permitted. However, permission to reprint/republish this material for advertising or promotional purposes or for creating new collective works for resale or redistribution must be obtained from the IEEE by writing to pubs-permissions@ieee.org. By choosing to view this document, you agree to all provisions of the copyright laws protecting it 


\title{
Modes of composite defects in 2D photonic crystals
}

\author{
K. B. Dossou ${ }^{1}$, L. C. Botten ${ }^{1}$, S. Mahmoodian², R. C. McPhedran², \\ C. G. Poulton ${ }^{1}$, A. A. Asatryan ${ }^{1}$ and C. Martijn de Sterke ${ }^{2}$ \\ Centre for Ultra-high-bandwidth Devices for Optical Systems \\ ${ }^{1}$ Department of Mathematical Sciences, University of Technology, Sydney, NSW 2007, Australia \\ Phone: 61-2-9514 2145, Fax: 61-2-9514 2260, E-Mail: Kokou.Dossou@uts.edu.au \\ ${ }^{2}$ School of Physics, University of Sydney, NSW 2006, Australia
}

\begin{abstract}
We demonstrate the existence of a class of defects in 2D photonic crystals, for which the eigenstates depend only on the defect geometry, and not on details like photonic crystal parameters or defect size.

\section{Introduction}

We study defect modes in 2D photonic crystals (PCs). Although many numerical studies have been undertaken, defects in the vicinity of band-edges are difficult to model accurately, since their fields are highly extended. Multiple defects are similarly difficult to handle. We develop a semi-analytic model for multiple defects based on an asymptotic expansion of the Green's function near a band edge and show that, for certain multiple defects, the eigenstates only depend on the geometry [1,2].
\end{abstract}

\section{Mode created by multiple defects}

We consider a 2D PC with a periodic dielectric constant $\varepsilon(\boldsymbol{r})$ and then change this in a finite number of unit cells to form a set of defects. By altering the difference between the new dielectric constant distribution $\tilde{\varepsilon}(\boldsymbol{r})$ and the original $\varepsilon(\boldsymbol{r})$, the defect modes sweep across the band gaps. Of particular interest are the ways in which the modes can behave near gap edges.

Our semi-analytic model is based on the analysis of the Green function $G\left(\boldsymbol{r}, \boldsymbol{r}^{\prime} ; \omega\right)$, representing the field at point $\boldsymbol{r}$ due to a source at point $\boldsymbol{r}^{\prime}$, and satisfying the inhomogeneous Helmholtz equation. We consider the situation where the defect mode frequency $\omega$ lies in a complete band gap and close to a gap edge of frequency $\omega_{L}$ which occurs for the Bloch vector $\boldsymbol{k}=\boldsymbol{k}_{L}$. For a single nondegenerate Bloch vector $\boldsymbol{k}_{L}$, we have $[1,2]$

$G\left(\boldsymbol{r}, \boldsymbol{r}^{\prime} ; \omega\right) \simeq \frac{c^{2} C_{L} K_{0}\left(b\left|\sigma\left(\boldsymbol{r}-\boldsymbol{r}^{\prime}\right)\right|\right)}{2 \pi \omega_{L} M_{L}} \psi_{L}\left(\boldsymbol{k}_{L}, \boldsymbol{r}\right) \psi_{L}^{*}\left(\boldsymbol{k}_{L}, \boldsymbol{r}^{\prime}\right)(1)$

where $\psi_{L}\left(\boldsymbol{k}_{L}, \boldsymbol{r}\right)$ is the Bloch mode at the band edge and $M_{L}=\left\langle\psi_{L}, \psi_{L}\right\rangle$. This leading-order approximation contains two key elements: an envelope function which decays exponentially with increasing distance from the source point, $K_{0}\left(b\left|\sigma\left(\boldsymbol{r}-\boldsymbol{r}^{\prime}\right)\right|\right)$, and a periodic combination of the band edge Bloch modes $\psi_{L}\left(\boldsymbol{k}_{L}, \boldsymbol{r}\right) \psi_{L}^{*}\left(\boldsymbol{k}_{L}, \boldsymbol{r}^{\prime}\right)$. By using Green's Theorem, we may derive the exact result for TM $\left(\mathrm{E}_{/ /}\right)$polarisation

$$
\psi(\boldsymbol{r})=-\frac{\omega^{2}}{c^{2}} \sum_{j^{\prime}=0}^{N-1} \int_{C_{j^{\prime}}} \delta \varepsilon\left(\boldsymbol{r}^{\prime}\right) G\left(\boldsymbol{r}, \boldsymbol{r}^{\prime} ; \omega\right) \psi\left(\boldsymbol{r}^{\prime}\right) d^{2} \boldsymbol{r}^{\prime}
$$

in which $\delta \varepsilon\left(\boldsymbol{r}^{\prime}\right)=\tilde{\varepsilon}\left(\boldsymbol{r}^{\prime}\right)-\varepsilon\left(\boldsymbol{r}^{\prime}\right)$. Near each defect cylinder $C_{j^{\prime}}$, we approximate $\psi(\boldsymbol{r}) \approx B_{j^{\prime}} \psi_{L}\left(\boldsymbol{k}_{L}, \boldsymbol{r}\right)$ and derive, using the Green's function (1) and Eq. (2),

$\left[\begin{array}{c}B_{0} \\ B_{1} \\ \vdots \\ B_{N-1}\end{array}\right]=\left[\begin{array}{cccc}\beta_{0,0} & \beta_{0,1} & \cdots & \beta_{0, N-1} \\ \beta_{1,0} & \beta_{1,1} & \cdots & \beta_{1, N-1} \\ \vdots & \vdots & \vdots & \vdots \\ \beta_{N-1,0} & \beta_{N-1,0} & \cdots & \beta_{N-1, N-1}\end{array}\right]\left[\begin{array}{c}B_{0} \\ B_{1} \\ \vdots \\ B_{N-1}\end{array}\right]$,

the eigenvalues and eigenvectors of which yield the dispersion equation and mode structure. The diagonal coupling coefficient (4) dominates at the band edge,

$\beta_{j, j}=-Q_{L} \log |\delta \omega| \int_{C_{j^{\prime}}} \delta \varepsilon\left(\boldsymbol{r}^{\prime}\right)\left|\psi_{L}\left(\boldsymbol{k}_{L}, \boldsymbol{r}^{\prime}\right)\right|^{2} d^{2} \boldsymbol{r}^{\prime}$,

where $Q_{L}=\omega_{L} C_{L} /\left(4 \pi M_{L}\right)$, while the off-diagonal coupling coefficients

$\beta_{j, j^{\prime}}=Q_{L} K_{0}\left(b\left|\sigma\left(\boldsymbol{o}_{j}-\boldsymbol{o}_{j^{\prime}}\right)\right|\right) \int_{C_{j^{\prime}}} \delta \varepsilon\left(\boldsymbol{r}^{\prime}\right)\left|\psi_{L}\left(\boldsymbol{k}_{L}, \boldsymbol{r}^{\prime}\right)\right|^{2} d^{2} \boldsymbol{r}^{\prime}$

are approximately the same as $\beta_{j, j}$ near the gap edge but are exponentially smaller, either deeper into the gaps, or when the defects are widely spaced, i.e., in the tight binding regime. Near the gap edge, $\beta_{j, j^{\prime}} \approx \beta_{0,0}$ (assum-

ing identical defects), and $\boldsymbol{B}=[1, \ldots, 1]^{T}$ is an eigenvector, with the dispersion equation for the corresponding defect (the fundamental mode) becoming $N \beta_{0,0}=1$, or

$$
\omega-\omega_{L}=A e^{-S /(N \delta \mathcal{E} / \mathcal{E})} .
$$

Eq. (6) mirrors the gap edge form [1] of the dispersion equation of a single defect mode, in which $\delta \mathcal{E} / \mathcal{E}$ denotes the relative change in the electrical energy caused by the defect, and $S$ is the defect inertia which is inversely proportional to the density of states.

For multiple defects $(N>1)$, the defects accumulate, increasing the slope of the dispersion curve of the fundamental mode. In addition other defect modes appear which are linked with the symmetry of the spatial arrangement of the defects. In Fig. 1 we show the evolution of the two defect modes in a double-defect PC. The matrix of the system (3) is symmetric and so the eigensolutions are $\boldsymbol{B}=[1,1]^{T}$ and $\boldsymbol{B}=[1,-1]^{T}$. The fundamental mode asymptotes exponentially towards the band edge, while the lower-frequency mode evolves along a trajectory which moves directly into the band, and is not strongly affected by it. We may understand this contrasting behaviour by comparing the field profiles (see Fig. 2) 


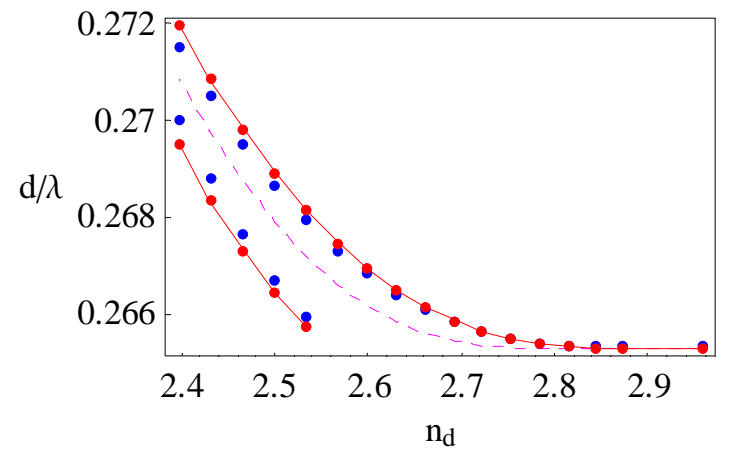

Fig. 1. Evolution of two double defect modes near the band edge for TM polarization, a square array with pitch $\mathrm{d}$, cylinder radius $0.3 \mathrm{~d}$; the unperturbed cylinders have refractive index $n=3$, while the two defect cylinders have index $\mathrm{n}_{\mathrm{d}}$ and separation $4 \mathrm{~d}$. Red curve: actual dispersion curve; Blue: analytic approximation; Magenta curve shows the dispersion curve for a single defect.

of the defect modes with that of the band-edge Bloch mode. We see that the high-frequency mode has the same symmetry (even) as the band-edge mode, which allows them to merge, while the lower mode has the opposite symmetry. The difference in behaviour is then seen to be that between avoided and normal crossing.
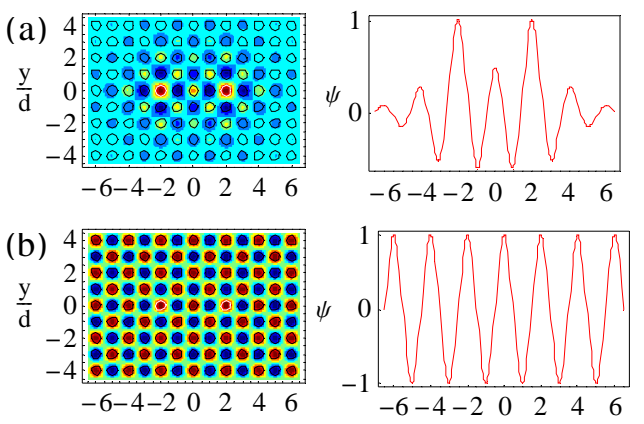

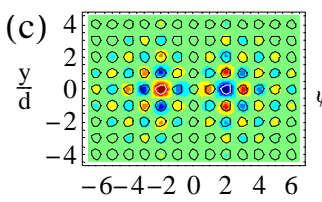

$\mathrm{x} / \mathrm{d}$

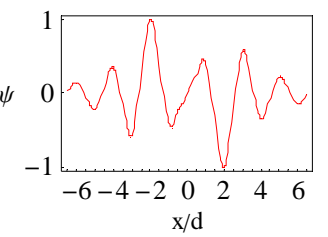

Fig. 2. Field profiles of (a) upper frequency mode (even symmetry), (b) Bloch mode for the perfect structure (even symmetry), and (c) lower frequency mode (odd symmetry).

For defect modes that do not have the symmetry of the band edge, we can, in fact, use the tight-binding model (which normally is regarded as applicable only near the centre of the band-gap) with reasonable accuracy near the band-edge. This allows us to provide semi-analytic estimates for the defect strength with which modes of this type enter the band.

\section{Circulant matrices}

When the centres of the defect cylinders form a regular polygon, then the matrix (3) of coupling coefficients takes the form of a circulant matrix. Such matrices have the property that their eigenvectors are independent of the particular values of the matrix elements. For the defect we are considering here this means that the eigenvectors, i.e., the defect state, depends only on the defect geometry, and not, for example, on the defect separation. This stability is a property usually only associated with a two-defect, but is here shown to generalize. (a)

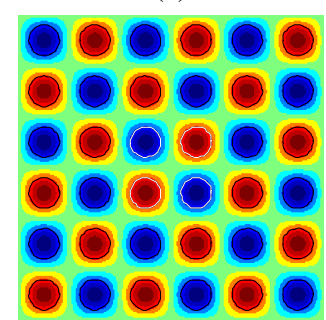

(c): $\mathrm{B}=[1,-1,-1,1]$

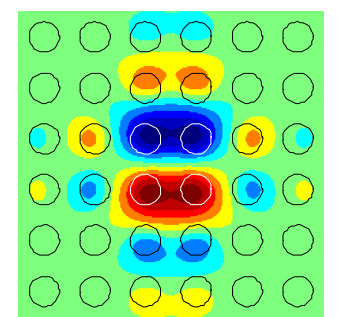

(b): $\mathrm{B}=[1,1,1,1]$

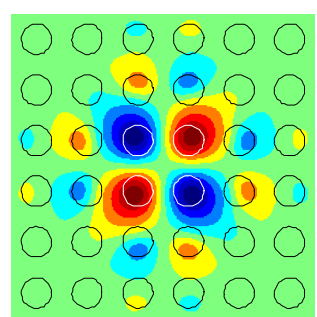

(d): $B=[1,-1,1,-1]$

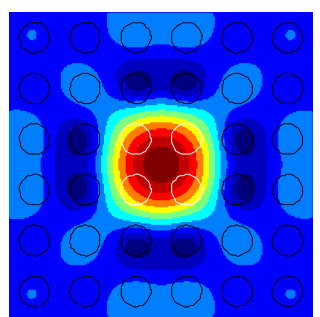

Fig 3. Eigenmodes for the square defect structure, with the same data as for Fig. 1. (a) shows the Bloch mode; panels (b) and (d) show singly degenerate modes, with the former being the fundamental mode; (c) shows one member of a degenerate pair, the other having $\mathrm{B}=[1,1,-1-, 1]$.

For four defects forming a square, for example, the $\beta$ matrix of Eq. (3) is

$$
\left[\begin{array}{llll}
\beta_{0} & \beta_{1} & \beta_{2} & \beta_{1} \\
\beta_{1} & \beta_{0} & \beta_{1} & \beta_{2} \\
\beta_{2} & \beta_{1} & \beta_{0} & \beta_{1} \\
\beta_{1} & \beta_{2} & \beta_{1} & \beta_{0}
\end{array}\right],
$$

the associated eigenvectors and eigenstates of which are illustrated in Fig. 3.

\section{Discussion and conclusions}

While the discussion here is for modes in square arrays for TM polarisation, the key result, namely that the eigenstates depend only on the defect geometry, and not on details such as PC parameters, defect size or polarisation, is quite general. For this reason, the spatial structure of the modes is quite stable across the gap, even though the coupling strength may vary strongly.

This work was carried out with the support of the ARC Centres of Excellence program.

\section{References}

1. K. B. Dossou et al, "Defect modes in two-dimensional photonic crystals,” Phys. Rev. A, submitted, (2008)

2. K. B. Dossou et al, Opt. Express 15, 4753 (2007)

3. S. Wilcox et al, Phys. Rev. E 71, 056606 (2005) 